\title{
Surface morphology evolution of a polycrystalline diamond by inductively coupled plasma reactive ion etching (ICP-RIE)
}

Yuting Zheng ${ }^{\mathrm{a}, \mathrm{b}}$, Haitao Ye ${ }^{\mathrm{b},}$, Jinlong Liu ${ }^{\mathrm{a}}$, Junjun $\mathrm{Wei}^{\mathrm{a}}$, Liangxian Chen ${ }^{\mathrm{a}}$, Chengming Li ${ }^{\mathrm{a}}{ }^{\text {* }}$

a. Institute for Advanced Materials and Technology, University of Science and Technology Beijing, Beijing 100083, PR China.

b. Department of Engineering, University of Leicester, Leicester, LE1 7RH, UK

E-mail address: nevermoreKEVIN@163.com,yz516@leicester.ac.uk

*Corresponding author

E-mail address: chengmli@mater.ustb.edu.cn, TEL: +(86)010 62332390

haitao.ye@leicester.ac.uk, TEL: +44(0)1162297377

Abstract: The needle-like surface morphology evolution in oxygen plasma in combination with a secondary gas $\left(\mathrm{Cl}_{2}, \mathrm{CHF}_{3}\right.$ or $\left.\mathrm{CF}_{4}\right)$ by inductively coupled plasma reactive ion etching (ICP-RIE) on a free-standing polycrystalline diamond was investigated. The addition of $\mathrm{CF}_{4}$ can produce trans-polyacetylene $(t$-PA), which is similar to the result when the pure $\mathrm{O}_{2}$ etching takes place, and generate compact needle-tip particles. However, the $t$-PA disappears with the introduction of $\mathrm{Cl}$ or $\mathrm{H}$ ions. The optimised etching parameters for the needle-like structure formation are as following: $\mathrm{Cl}_{2} / \mathrm{O}_{2}$ ratio $20 \%$ and $\mathrm{RF}$-power (RFP) $100 \mathrm{~W}$, where more compact and even nano-needles are realised with an average etching rate of $2 \mu \mathrm{m} / \mathrm{min}$. The $\mathrm{Cl}_{2} / \mathrm{O}_{2}$ plasma etching results indicate that the time-dependent etching mechanism of diamond nano-needles results from (111) crystal plane selective etching and preferential graphitisation at the twin-plane boundary and dislocation area.

Key words: Polycrystalline diamond, plasma etching, X-ray techniques, Raman, Structural,

\section{Introduction}

The emergence of chemical vapor deposition (CVD) techniques for the synthesis of diamond thin films has resulted in overwhelming interest in the use of this material for a wide range of electronic device applications. The focus of most electronic device studies to date has been the fabrication of high-performance power transistor devices, microelectromechanical systems (MEMS), electron field emitting devices, and nitrogen-vacancy center based photonic and quantum devices [1-4]. One of key challenges to fabricate such devices is the ability to control and obtain a specific diamond surface for the subsequent process and application purpose.

Reactive ion etching (RIE) is an effective process for the formation of diamond nanostructures with precise and controllable dimension. Particularly for inductively coupled plasma (ICP)-RIE, it delivers higher etching rate, better uniformity, higher selectivity and lower crystal lattice damage. Pure oxygen plasma has been used to produce diamond micro-mechanical components, whilst the majority of etching processes are based on 
oxygen plasma in combination with a secondary gas [5-6]. There are a number of reports on the diamond nano-structuring by RIE. I-N Lin et al. have reported the fabrication of nano-needles on ultra-nanocrystalline diamond by RIE [4]. Izak et al. have introduced ICP-RIE to realize the needle-like formation on polycrystalline diamond (PCD) [7]. Practically, the characteristics (e.g. fabricated colour centre or ultra-high elastic deformation) of such needle-like structures offers the new potential for applications, such as single photon sources or strain-mediated nano-mechanical resonators, etc, through optimised design of diamond nanostructure $[4,8]$. However, there are few reports on the systematic studies of the formation of the nanostructures against auxiliary gas effect and the associated diamond surface evolution process.

Here, the optimisation and mechanism of nano-needle formation on PCD in different gas system were studied. The PCD with large grain size of over 200 um was selected for this study, in order to minimize the influence of grain boundaries

\section{Experiments}

The used PCDs $5 \times 5 \times 1 \mathrm{~mm}^{3}$ in size, cut from a 120 -mm-diameter free-standing PCD wafer, which was deposited for $200 \mathrm{~h}$ at $900{ }^{\circ} \mathrm{C}$ with a rate of $7.5 \mu \mathrm{m} / \mathrm{h}$ by a home-built $100 \mathrm{~kW}$ DC arc-jet plasma CVD. To ensure the excellent uniformity of the samples, the entire PCD wafers were polished for one week. These samples were treated in boiling mixed acids and then cleaned in acetone and methanol. Dry etching was carried out using an ICP-RIE system (SENTECH/SI-500) with steady coil power of $800 \mathrm{~W}$. Various etching processes and related parameters are summarized in Table 1.

Table 1. Diverse ICP-RIE processes and related parameters

\begin{tabular}{cccccc}
\hline No. & $\begin{array}{c}\text { Gas components } \\
(\mathbf{\%})\end{array}$ & $\mathbf{R F P}(\mathbf{b i a s})$ & $\begin{array}{c}\text { Etching time } \\
(\mathbf{m i n})\end{array}$ & $\begin{array}{c}\text { Etched } \\
\text { thickness }(\boldsymbol{\mu m})\end{array}$ & $\begin{array}{c}\text { Etching rate } \\
(\boldsymbol{\mu m} / \mathbf{m i n})\end{array}$ \\
\hline PCD1 & $\mathrm{O}_{2}=100$ & 100 & 5 & 7 & 1.4 \\
PCD2 & $\mathrm{O}_{2}=100$ & $100+400$ & $5+5$ & - & - \\
PCD3,4,5 & $\mathrm{Cl}_{2} / \mathrm{O}_{2}=5,10,20$ & 100 & 5 & $8,6,10$ & $1.6,1.2,2$ \\
PCD6,7,8 & $\mathrm{CHF}_{3} / \mathrm{O}_{2}=5,10,20$ & 100 & 5 & $4,11,18$ & $0.8,2.2,3.6$ \\
PCD9,10,11 & $\mathrm{CF}_{4} / \mathrm{O}_{2}=5,10,20$ & 100 & 5 & $6,17,7$ & $1.2,3.4,1.4$ \\
PCD12 & $\mathrm{Cl}_{2} / \mathrm{O}_{2}=10$ & 100 & $5+5+5$ & 19 & 1.3 \\
\hline
\end{tabular}

A scanning electron microscope (SEM-HITACHI-S4800), atomic force microscopy (AFM, ASYLUM-HVA220) were used to observe the morphology. The etching rates, which were in line with the time reported [7], were estimated using ultra-precise profiler measurements (GUANGLU) by testing the thickness before and after etching. Furthermore, the phases were demonstrated via Raman spectroscopy (NANOPHOTO) with a $532 \mathrm{~nm}$ excitation wavelength and the crystal orientations of PCDs were investigated by X-ray 
diffractometer (XRD-Rigaku). Supportively, the surface chemical bonds of the samples were further characterized by X-ray photoelectron spectroscopy (XPS, Thermo Scientific, ESCALAB-250Xi). Before fitting the curves, the spectrum results of XPS were calibrated by the adventitious carbon binding energy [9]

\section{Results and Discussion}
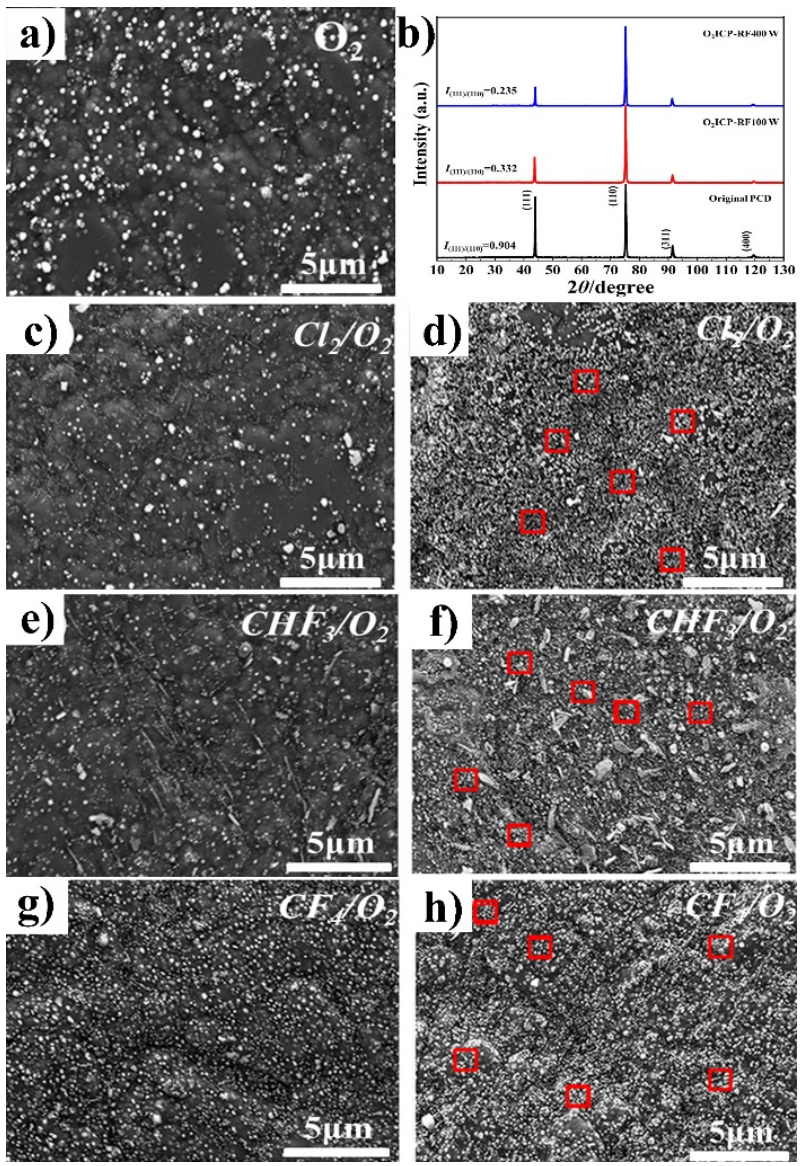

Figure.1. Surface morphology of etched samples: a) PCD1; c) PCD4; d) PCD5; e) PCD7; f) PCD8; g) PCD10, h) PCD11, and the effect of $\mathrm{O}_{2}$ : b) XRD patterns of multistep etched PCD2.

Figure. 1 shows SEM images of various etched PCDs and XRD patterns of the original and pure $\mathrm{O}_{2}$ etched PCD2. In each $1-\mu \mathrm{m}^{2}$ randomly selected red square area, the number of the nano-needles can be counted statistically via the visible needle tips. In Figure.1(d), the average number of needles is approximately 42; whilst this value becomes 27 in Figure.1(f) and 28 in Figure.1(h), respectively. Meanwhile, the calculated standard deviations were $9.7 \%, 32.5 \%$, and $19.1 \%$, respectively. When oxygen was used alone as shown in Figure.1(a), the diamond surface presented wrinkled and creased morphologies, and nanoscale dots exhibited an uneven distribution with varying sizes. While the oxidation reaction of gas mixture plasma on diamond was related to the etched morphology, the auxiliary gas can also exerted extra effect during surface machining, e.g., etching rate. In Figure.1(c), several smaller nanoscale dots appeared when the $10 \% \mathrm{Cl}_{2}$ was added during the RIE process. Once $20 \%$ of $\mathrm{Cl}_{2}$ was added, a high volume of compact and homogeneous nano-needles appeared as 
shown in Figure.1(d). This was due to the accelerated etching rate $(2 \mu \mathrm{m} / \mathrm{min})$ and enhanced selective etching by higher concentration of $\mathrm{Cl}$. When $10 \% \mathrm{CHF}_{3}$ was added into the RIE chamber, more nano-needle tips appeared with some tendency for alignment as shown in Figure.1(e). Furthermore, when $20 \% \mathrm{CHF}_{3}$ was added, more nano-needle tips appeared but homogeneity deteriorated, which corresponds to the highest standard deviation of $32.5 \%$ described earlier. This was the result of the boosted etching rate $(3.6 \mu \mathrm{m} / \mathrm{min})$, which is likely due to the involvement of the $\mathrm{H}$ ions. However, when $10 \%$ and $20 \%$ of $\mathrm{CF}_{4}$ were added (instead of $\mathrm{CHF}_{3}$ ) to avoid the presence of $\mathrm{H}$ ions, as shown in Figure.1(g) and (h), some more dense and uniform needle-tips formed. Due to the relative faster etching rate $(3.4 \mu \mathrm{m} / \mathrm{min})$, in the $10 \% \mathrm{CF}_{4}+\mathrm{O}_{2}$ environment, the nano-needles were more apparent and the needle surfaces appeared fresh and sharp-edged. Nevertheless, $20 \%$ addition of $\mathrm{CF}_{4}$ negatively impacted the etching rate (down to $1.4 \mu \mathrm{m} / \mathrm{min}$ ). In addition, figure.1(b) shows the XRD patterns of the original and $\mathrm{O}_{2}$ plasma etched PCD2. After etching at RFP of $100 \mathrm{~W}, I_{(111) /(110)}$, which is the peak intensity ratio of the (111) and (110) orientation, decreases from 0.904 to 0.332 . This demonstrates that the (111) crystal plane of etched PCD exhibits weaker intensity and becomes less dominant in XRD diffraction content after etching. This was due to preferential etching/oxidation of the (111) orientation compared with other crystal plane, which agrees with existing literature [7,10]. However, after subsequent etching at RFP of $400 \mathrm{~W}, I_{(111) /(110)}$ decreases slightly from 0.332 to 0.235 . This minor change occurred owing to the dominant role of ion-bombardment enhanced etching when the RFP was as high as $400 \mathrm{~W}$, which surpassed the reactive selective etching on crystal orientations [11].
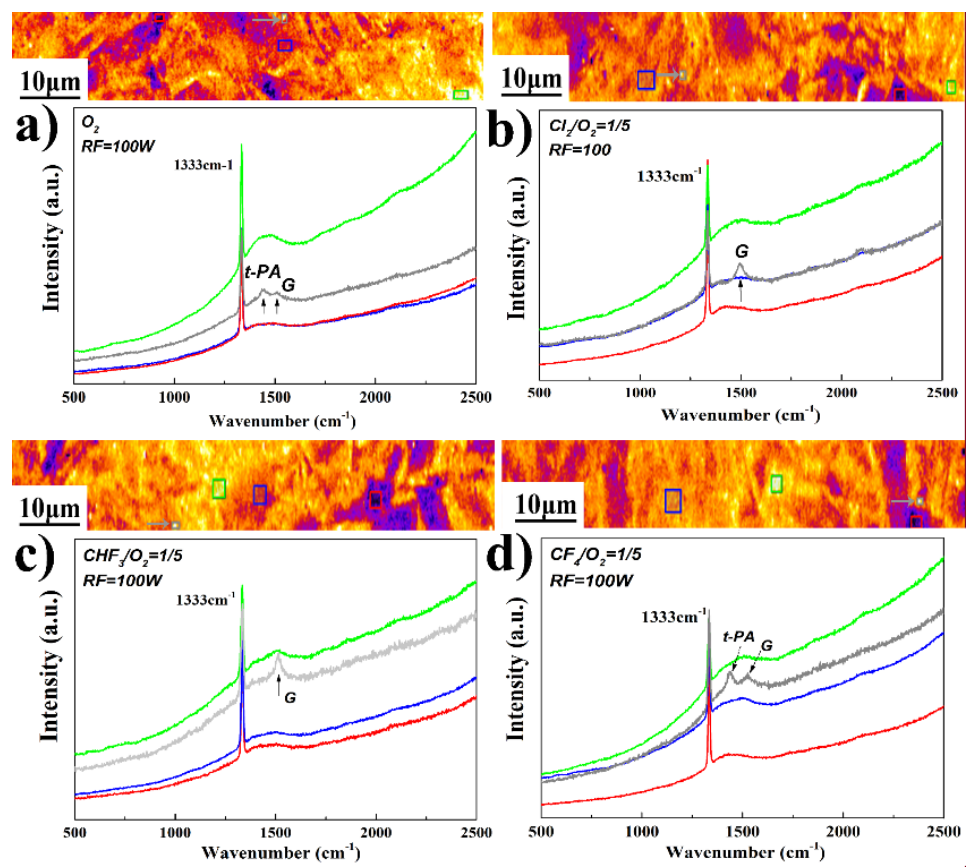

Figure.2. Raman mapping with different selected areas and corresponding spectra of the etched PCDs: a) PCD1; b) PCD5; c) PCD8; d) PCD11. 

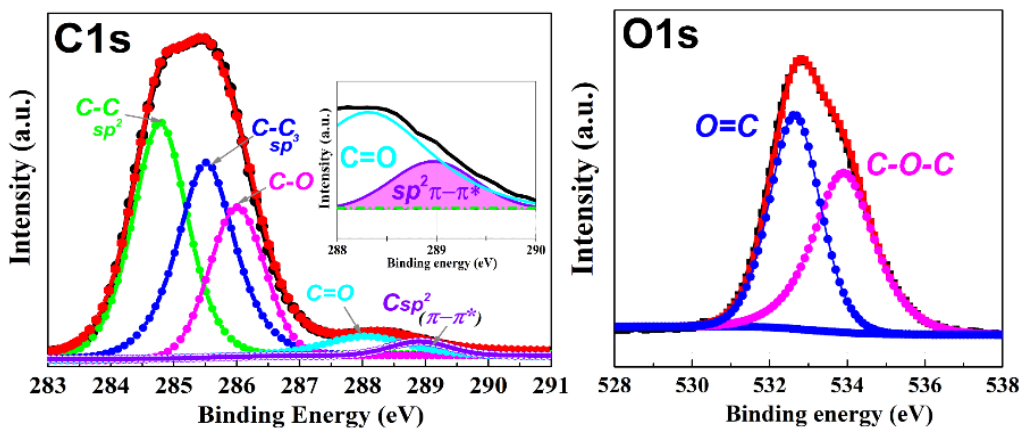

Figure.3. Fine fitted $C$ 1s and $O$ 1s XPS spectra of etched surface of PCD1. The inset is the amplified XPS shoulder peak assignment of $\pi$ - $\pi^{*}$ bonds.

To investigate the effect of auxiliary gas, we studied Raman spectroscopy on etched PCD samples with the highest additive amount. In Figure.2, the lines correspond to the same color square marked areas in the mapping pictures. These areas were specially selected from different color regions in the mapping images. We selected the white dots gathered parts of the SEM in mapping pictures, which were the transient state of the needle cluster, in a grey square frame. It should be mentioned that this transient state is the modality during the dynamic formation process from the needle-tip particle emergence to the intact diamond needle formation. In Figure.2(a) and (d), two distinct peaks of grey spectrum appeared at 1470 and $1580 \mathrm{~cm}^{-1}$, which attribute to the $\mathrm{C}=\mathrm{C}$ bonds of trans-polyacetylene ( $t$-PA) and $s p^{2}$ graphitic phases, respectively [12]. Thus the addition of $\mathrm{CF}_{4}$ made no difference on the Raman spectra compared with the samples etched in the pure $\mathrm{O}_{2}$ (the main gas source) environment. However, in Figure.2(b) and (c), the disappearance of the $1470 \mathrm{~cm}^{-1}$ peaks may be due to the etching effect of $\mathrm{Cl}$ and $\mathrm{H}$. This can be interpreted as that the $\pi$ bond of $t$-PA breaks when the $\mathrm{H}$ (or $\mathrm{Cl}$ ) atoms cover the dangling bonds of the surface $\mathrm{C}$ atoms of etched ditches [13]. Furthermore, the peaks of $s p^{2}$ graphitic bonds in Figure.2(c) and (d) were sharper, because of the synergetic oxidation effect of the F element on diamond phase and the self-contained $\mathrm{C}$ element. The bulge for the rest of the spectra from 1450 to $1580 \mathrm{~cm}^{-1}$ and the obvious background spectra resulted from the disorder or dislocation such as nano-needles and rugged regions where with amorphous carbon. Figure.2(b) illustrates that these four coloured lines are almost identical, indicating that the etched diamond quality in different regions is the same. Combining with SEM images, the optimal nano-needle formation recipe is the addition of $20 \%$ of $\mathrm{Cl}_{2}$ into $\mathrm{O}_{2}$. Meanwhile, figure. 3 shows the deconvoluted high-resolution XPS spectra of PCD1, which etched in the pure $\mathrm{O}_{2}$ plasma environment. It is observed that the C 1s peak can be mainly deconvoluted into four components at $284.6,285.5,286.2$ and 288.1 $\mathrm{eV}$, which are attributed to $s p^{2}$ graphitic phases, $s p^{3}$ diamond phase, $\mathrm{C}-\mathrm{O}$ and $\mathrm{C}=\mathrm{O}$ bonds, respectively [12-14]. It should be noted that in the C1s core-energy level spectrum of sample, a shoulder peak at about $289 \mathrm{eV}$ is related to $\pi-\pi^{*}$ bonds and considered as the characteristic of the $\mathrm{C}=\mathrm{C}$ bonds of $t-\mathrm{PA}$, which is shown by the Raman assignment at $1470 \mathrm{~cm}^{-1}$ in Figure.2(a) (it also be the same to this peak in Figure.2(d)) [12,13]. 
Furthermore, the deconvoluted $\mathrm{O} 1 \mathrm{~s}$ peaks at 532.4 and $533.9 \mathrm{eV}$ are attributed to the $\mathrm{O}=\mathrm{C}$ and $\mathrm{C}-\mathrm{O}-\mathrm{C}$ bonds of $s p^{2}$ graphite, respectively, which was generated from oxidation (reactive etching) of diamond surface [14].

Besides the selective etching of different crystal orientations, preferential etching of the dislocation and boundary area also plays an important role in the diamond-needle formation. Figure.4 presents the nano-needle formation mechanism with the $\mathrm{Cl}$ addition at a small flow rate, at which $\mathrm{PCD}$ was etched slower than the optimized process so that the step-by-step investigation was presented easily. A smooth surface (RMS:1.59 nm) of as-polished PCD was shown with light-scratches and grain size larger than $200 \mu \mathrm{m}$ in Figure.4(a, $\left.\mathrm{a}_{1-2}\right)$. These large grains can act as a homogeneous unit. After $5 \mathrm{~min}$ etching, in Figure.4(b), the cauliflower-like morphologies can be seen, which consist of some smaller pieces and etched boundary. Meanwhile, the preferential etching of the dislocation area and the twin boundary revealed a sand grain-shaped particle, twin, four-leaf clover and cauliflower shapes among some of the slowly etched parts (200-500 nm). This indicates a nano-needle transient state in which the twin boundary and the planar defects were etched preferentially in addition to the etchings on the cauliflower-shaped parts. The nano-needles originate from these parts and undergo further etching. During this process, the nearby regions also generate new particles and the process continues. This phenomenon is demonstrated in Figure.4(c), which shows much more compact needles (the width is less than $50 \mathrm{~nm}$ ). When the etching time was extended to $15 \mathrm{~min}$, as shown in Figure.4(d), many more even and tiny nano-needles (the width is less than $10 \mathrm{~nm}$ ) appeared. From the fine perspective of 3D images and automatic calculation results of average height shown in Figure.4( $\left.b_{1}, c_{1}, d_{1}\right)$, the sample surface changed from its initial rugged features to the newly generated needles with statistical mean length (SML) of $41.54 \mathrm{~nm}$ and then to the full-fledged nano-needles with SML of $56.52 \mathrm{~nm}$. Consequently, the average high aspect ratio (large than $5: 1)$ of the nano-needles was obtained.

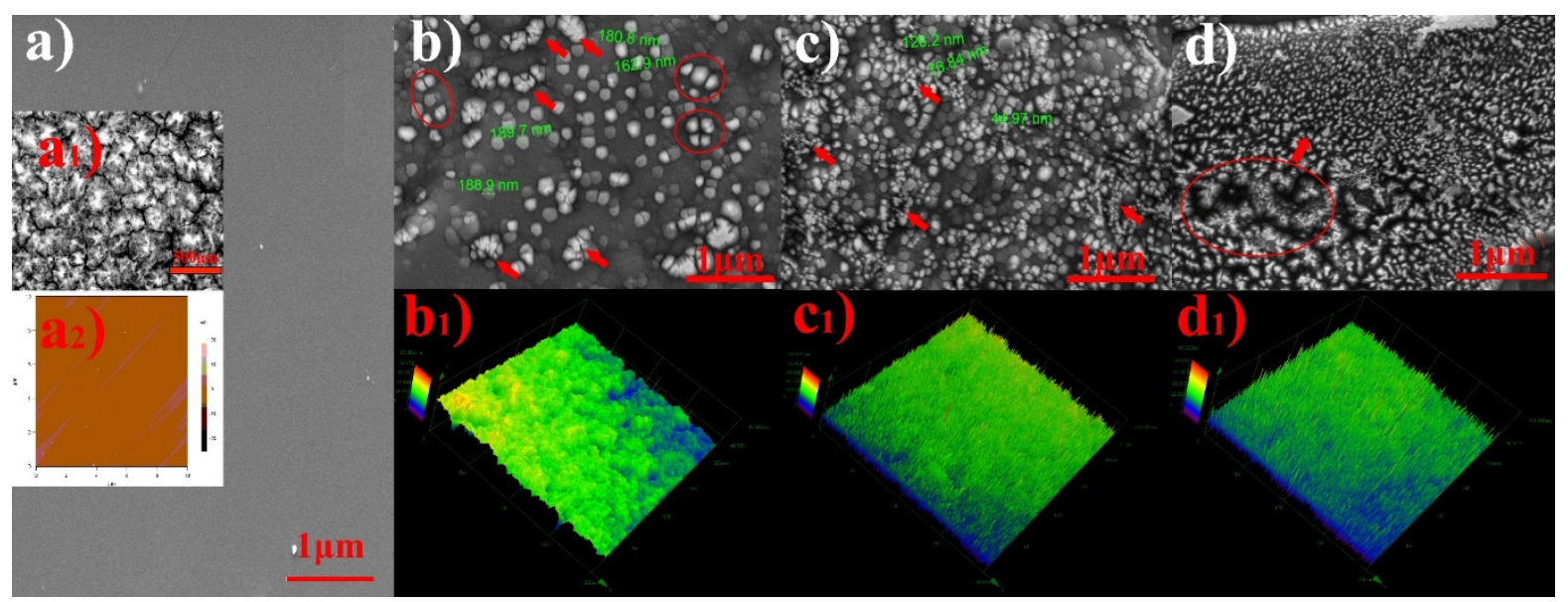

Figure.4. Surface morphology of original PCD and time-dependent etching results: a) SEM, $\left.\mathbf{a}_{1}\right) \mathrm{OM}, \mathrm{a}_{2}$ ) AFM images of as-polished original PCD12; SEM and 3D-AFM images of PCD12 etched for different duration: b) and $b_{1}$ ) of 5 mins; c) and $c_{1}$ ) of $10 \mathrm{mins}$; d) and $d_{1}$ ) of 15 mins. 


\section{Conclusion}

The evolution of the needle-like structure formation by ICP-RIE on free-standing PCDs, were investigated using step-by-step morphology deduction and phase analysis. The $\mathrm{CF}_{4} / \mathrm{O}_{2}$ plasma can produce the compact needle-tip particles and generate the chemical bonds associated with trans-polyacetylene. However, both adding $\mathrm{Cl}_{2}$ and $\mathrm{CHF}_{3}$ in $\mathrm{O}_{2}$ plasma can destroy the trans-polyacetylene. An optimised process condition for obtaining more compact and uniform nano-needle structure is to use $\mathrm{Cl}_{2} / \mathrm{O}_{2}=20 \%$ plasma, and RFP of $100 \mathrm{~W}$ with a resultant etching rate of $2 \mu \mathrm{m} / \mathrm{min}$. The physical mechanism associated with the observed phenomenon can be explained by the time-dependent etching of diamond nano-needles under low plasma power where the synergetic effect between the crystal orientation selective etching and the preferential oxidation at the twin-plane boundary and dislocation regions plays a dominant role.

\section{Declaration of Competing Interest}

None

\section{Acknowledgment}

This work was supported by the National Key Research and Development Program of China (no. 2016YFE0133200) and the European Union's Horizon-2020 Research program (no. 734578). Special thanks to the national high-level university-sponsored graduate program of China Scholarship Council (CSC).

\section{References}

[1] S. Shikata, Single crystal diamond wafers for high power electronics, Diam. Relat. Mater., 65 (2016) 168-175. https://doi: 10.1016/j.diamond.2016.03.013.

[2] J. Isberg, J. Hammersberg, E. Johansson, T. Wikström, D.J. Twitchen, A.J. Whitehead, S.E. Coe, G.A. Scarsbrook, High carrier mobility in single-crystal plasma-deposited diamond, Science, 297 (2002) 1670. https://doi.org/10.1126/science.1074374.

[3] R.S. Sussmann, CVD Diamond for Electronic Devices and Sensors. John Wiley \& Sons, (2009).

[4] S. Kunuku, K.J. Sankaran, C.Y. Tsai, W. H. Chang, N.H. Tai, K.C. Leou, I.N. Lin, Investigations on diamond nanostructuring of different morphologies by the reactive ion etching process and their potential applications, ACS. Appl. Mater. Interfaces., 15 (2013) 7439-7449. https://doi.org/10.1021/am401753h.

[5] Y. Tao, C. L. Degen, Single-crystal diamond nanowire tips for ultrasensitive force microscopy. Nano. Lett., 15 (2015) 7893-7897. https://doi.org/10.1021/acs.nanolett.5b02885.

[6] A. Toros, M. Kiss, T. Graziosi, H. Sattari, P. Gallo, N. Quack, Precision micro-mechanical components in single crystal diamond by deep reactive ion etching, Microsyst. Nanoeng., 12 (2018) 1-8. 
https://doi.org/10.1038/s41378-018-0014-5.

[7] T. Izak, A. Kromka, O. Babchenko, M. Ledinskya, K. Hruska, E. Verveniotis, Comparative study on dry etching of polycrystalline diamond thin films, Vacuum, 86 (2012) 799-802. https://doi.org/10.1016/j.vacuum.2011.07.023.

[8] A. Banerjee, D. Bernoulli, H. Zhang, M.F. Yuen, J.B. Liu, J.C. Dong, F. Ding, J. Lu, M. Dao, W.J. Zhang, Y. Lu, S. Suresh, Ultra-large elastic deformation of nanoscale diamond, Science, 360 (2018) 300-302. https://doi.org/10.1126/science.aar4165.

[9] B.V. Crist, XPS in industry_-Problems with binding energies in journals and binding energy databases, J. Electron. Spectrosc. Relat. Phenom., 231 (2019) 75-87. https://doi.org/10.1016/j.elspec.2018.02.005.

[10] C.Q. Sun, H. Xie, H.T. Ye, P. Hing, Preferential oxidation of diamond $\{111\}$, J. Phys. D: Appl. Phys., 33 (2000) 2196-2199. https://doi.org/10.1088/0022-3727/33/17/316.

[11] M.J. Jackson, W. Ahmed, Micro and Nano-manufacturing, volume II. Springer, 2018.

[12] X.J. Hu, C.K. Chen, S.H. Lu, High mobility n-type conductive ultra-nanocrystalline diamond and graphene nanoribbon hybridized carbon films, Carbon, 98 (2016) 671-680. https://doi.org/10.1016/j.carbon.2015.11.057.

[13] S.J. Sque, R. Jones, P.R. Briddon, Structure, electronics, and interaction of hydrogen and oxygen on diamond surfaces, Phys. Rev. B., 73 (2006) 085313. https://doi.org/10.1103/PhysRevB.73.085313.

[14] R.A. Gaashani, A. Najjar, Y. Zakaria, S. Mansoura, M.A. Atieha, XPS and structural studies of high quality graphene oxide and reduced graphene oxide prepared by different chemical oxidation methods, Cream. Int., 45 (2019) 14439-14448. https://doi.org/10.1016/j.ceramint.2019.04.165. 